\title{
Covering All the Bases: Complementary MR1 Antigen Presentation Pathways Sample Diverse Antigens and Intracellular Compartments
}

\author{
Corinna Kulicke ${ }^{1,2}$, Elham Karamooz ${ }^{1,2}$, David Lewinsohn ${ }^{1,2,3,4}$ and Melanie Harriff ${ }^{1,2,4 *}$ \\ ${ }^{1}$ Pulmonary and Critical Care Medicine, Oregon Health and Science University, Portland, OR, United States, ${ }^{2}$ VA Portland \\ Health Care System, Research and Development, Portland, OR, United States, ${ }^{3}$ Department of Pediatrics, Oregon Health \\ and Science University, Portland, OR, United States, ${ }^{4}$ Department of Molecular and Microbial Immunology, Oregon Health \\ and Science University, Portland, OR, United States
}

\section{OPEN ACCESS}

Edited by:

Ildiko Van Rhijn,

Brigham and Women's Hospital and

Harvard Medical School,

United States

Reviewed by:

Hamish McWilliam,

The University of Melbourne, Australia

David H. Margulies,

National Institutes of Health $(\mathrm{NIH})$,

United States

*Correspondence:

Melanie Harriff

harriffm@ohsu.edu

Specialty section:

This article was submitted to Antigen Presenting Cell Biology,

a section of the journal

Frontiers in Immunology

Received: 16 April 2020

Accepted: 27 July 2020

Published: 02 September 2020

Citation:

Kulicke C, Karamooz E, Lewinsohn D and Harriff M (2020) Covering All the Bases: Complementary MR1 Antigen

Presentation Pathways Sample Diverse Antigens and Intracellular Compartments.

Front. Immunol. 11:2034.

doi: 10.3389/fimmu.2020.02034
The ubiquitously expressed, monomorphic MHC class Ib molecule MHC class I-related protein 1 (MR1) presents microbial metabolites to mucosal-associated invariant T (MAIT) cells. However, recent work demonstrates that both the ligands bound by MR1 and the $\mathrm{T}$ cells restricted by it are more diverse than originally thought. It is becoming increasingly clear that MR1 is capable of presenting a remarkable variety of both microbial and non-microbial small molecule antigens to a diverse group of MR1-restricted T cells (MR1Ts) and that the antigen presentation pathway differs between exogenously delivered antigen and intracellular microbial infection. These distinct antigen presentation pathways suggest that MR1 shares features of both MHC class I and MHC class II antigen presentation, enabling it to sample diverse intracellular compartments and capture antigen of both intracellular and extracellular origin. Here, we review recent developments and new insights into the cellular mechanisms of MR1-dependent antigen presentation with a focus on microbial MR1T cell antigens.

Keywords: antigen presentation, MR1, MAIT cell, ligands, endosomal trafficking

\section{INTRODUCTION}

The immune system is traditionally thought of as dichotomous. On the one hand, the innate immune system is activated by broadly conserved pathogen-associated molecular patterns (PAMPs) detected by germline-encoded pattern recognition receptors. By contrast, the adaptive immune response relies on somatic re-arrangement of antigen receptor genes to generate the diversity and specificity needed to sense extensively processed peptide antigens in the context of highly polymorphic major histocompatibility complex (MHC) molecules. However, it is increasingly appreciated that these categories rather represent the extremes of a spectrum with non-classical immune cell subsets such as innate-like, donor-unrestricted T cells (DURTs) defying a binary classification (1-3). Instead, DURTs have attributes of both adaptive and innate immunity. For example, while they express somatically re-arranged T cell receptors (TCRs), their TCR repertoire is limited, and in many cases semi-invariant. Moreover, although these TCRs recognize their cognate antigen in the context of antigen presenting molecules, they are restricted by highly conserved, monomorphic proteins displaying primarily non-peptidic ligands $(1,3)$. One of these is 
the MHC class Ib molecule MHC I-related protein 1 (MR1). First identified in 1995 as an MHC I-related gene encoded outside the MHC locus (4), MR1 was later found to be the restricting element of the innate-like mucosal-associated invariant T (MAIT) cells (5). Like other non-conventional T cell subsets, these cells express a limited TCR repertoire and rapidly exert effector functions upon activation [recently reviewed in (6)]. While classical MAIT cells are defined by expression of the TRAV1-2 TCR $\alpha$ chain, more recent work has identified TRAV1-2 ${ }^{-}$T cells that are activated in an MR1-dependent manner, expanding the family of MR1-restricted T cells (MR1Ts) (5, 7-11). The first MAIT cellactivating MR1 ligands identified were intermediates produced during the microbial biosynthesis of riboflavin (vitamin B2) (12, 13). Since mammalian cells do not express the enzymes of this biosynthetic pathway, riboflavin precursors represent a microbederived molecular pattern (14). Ongoing ligand identification efforts have revealed many more microbial and non-microbial MR1 ligands which comprise both agonists and antagonists of MR1T cell activation $(9,15-17)$. Importantly, the number of microorganisms that synthesize riboflavin or other putative MR1 ligands is large and includes many commensal species in addition to pathogens $(18,19)$. This, together with the frequency of MR1Ts and the ubiquitous expression of MR1, likely necessitates tight regulation of MR1 antigen presentation to prevent inappropriate activation (20).

Classical peptide antigen presentation relies on a division of labor on the molecular scale: ER-resident MHC class I molecules bind and present peptides derived from intracellular protein synthesis whereas MHC class II molecules survey endosomal compartments where they encounter extracellular material taken up by endo- or phagocytosis. Although there are exceptions to this paradigm such as cross-presentation of exogenous or particulate antigen on MHC class I molecules, broadly speaking, immune surveillance of endogenous and exogenous peptide antigen is achieved by compartmentalization of two different antigen presenting molecules with distinct intracellular trafficking patterns (21). By contrast, MR1 is the only known metabolite-presenting molecule, placing the burden of sampling both intracellular and exogenous sources of antigenic metabolites on a single molecule. Accordingly, it is becoming increasingly clear that MR1T antigens are presented through multiple specialized presentation mechanisms likely reflecting the biochemical properties as well as the abundance and intracellular distribution of the antigen $(20,22)$.

In this review, we will discuss recent advances in our understanding of both the expanding repertoire of MR1 ligands and current models for distinct pathways by which these ligands are presented to MR1Ts.

\section{TOWARD DEFINING THE MR1 LIGANDOME}

\section{Canonical MAIT Cell Antigens: Lumazines, Pterins, and Pyrimidine Neoantigens}

The identification of microbial riboflavin metabolism as a source of MAIT cell-activating ligands marked a major breakthrough for the MR1 field (12, 13). The first microbial MAIT cell antigens to be identified were ribityllumazine metabolites upstream of riboflavin (vitamin B2) biosynthesis. These MR1 ligands include 6,7-dimethyl-8-D-ribityllumazine (DMRL), 7 hydroxy-6-methyl-8-D-ribityllumazine (HMRL), and reduced 6-hydroxymethyl-8-D-ribityllumazine (rRL) (13). In the same report, Kjer-Nielsen et al., described the MAIT antagonist ligand 6-formylpterin (6-FP), which derives from folate (vitamin B9). 6-FP has the same bicyclic ring structure as the ribityllumazines but lacks the ribityl tail, which is critical for recognition by the MAIT TCR (23). A subsequent report described the formation of the pyrimidine neoantigens 5-(2-oxopropylidenamino)6-D-ribitylaminouracil (5-OP-RU) and 5-(2oxoethylideneamino)-6-D-ribitylaminouracil (5-OE-RU), which form upon the spontaneous reaction of the riboflavin precursor 5-aminoribityluracil (5-A-RU) with methylglyoxal or glyoxal, respectively (12). 5-OP-RU and 5-OE-RU have a single ring structure, but still feature the ribityl moiety. In a unique mode of antigen binding, both 6-FP and the pyrimidine neoantigens covalently bind to MR1 by forming a Schiff base with lysine residue 43 (K43) at the bottom of the MR1 antigen binding groove $(12,13)$. The ribityllumazine ligands, on the other hand, non-covalently associate with MR1, which correlates with lower antigenicity $(12,16,24)$. More recently, we identified the additional MR1T-activating ribityllumazine ligands photolumazine I (PLI) and photolumazine III (PLIII), as well as the antagonistic ligand 7,8-didemethyl-8-hydroxy-5deazariboflavin (FO) (15). All of these ligands could have 5A-RU as a precursor metabolite, suggesting that it may be a key molecule in the synthesis of MR1T antigens. In support of this, modulation of riboflavin biosynthesis correlates with MR1T activation (25) and deletion of the enzyme responsible for the synthesis of 5-A-RU abrogates MR1T recognition of some microbes $(12,13,25,26)$. Microbial vitamin B metabolites comprise the most potent and well-characterized MAIT cell agonists to date. However, recent studies described below clearly demonstrate that a much broader range of small molecule metabolites can bind to MR1 and activate MR1Ts.

\section{Beyond Vitamin B Metabolites: Evidence for Additional MR1 Ligands}

Aspects of both MR1 itself and the MR1T TCRs support the hypothesis that the MR1 ligand repertoire includes other classes of molecules in addition to those in the vitamin B family. MR1 is structurally similar to other MHC class I molecules in that its heavy chain consists of three extracellular domains $\left(\alpha_{1}-\alpha_{3}\right)$, a transmembrane domain, and a small cytosolic tail. Like MHC class Ia, MR1 non-covalently associates with $\beta_{2}$-microglobulin $\left(\beta_{2} \mathrm{~m}\right)$ to form a heterodimer (27). The MR1 antigen binding cleft is formed by the $\alpha 1$ and $\alpha 2$ domains of the heavy chain and consists of an A' and an F'-pocket $(23,24,28,29)$. The canonical antigens described above bind in the A'-pocket, which consists primarily of hydrophobic amino acids $(13,28)$. The non-polar nature of these residues accommodates organic ligands such as the vitamin B metabolites (30) and there are various other classes of small molecules with chemical properties consistent with 
binding in this groove $(15,16,30)$. Furthermore, the unoccupied space remaining in the MR1 ligand binding groove outside of the A' pocket leaves open the possibility for binding of additional ligands or chaperones (31). Recent advances in defining the TCRs restricted by MR1 support this notion. MAIT cells were originally defined by their semi-invariant TCR, which consists of the TRAV1-2 $\alpha$ chain paired with a limited number of $\beta$ chains, and features a signature CDR $3 \alpha$ sequence (11). However, numerous studies continue to expand the MR1T TCR repertoire [reviewed in: $(6,32)]$. While many of the MR1Ts with non-canonical MAIT TCRs recognize the vitamin B metabolite ligands, there are others that do not $(9,10,33)$. Additionally, even among those TCRs that do recognize vitamin $B$ metabolites, there is differential recognition of individual ligands by distinct TCRs $(7,15,34)$. Combined with the conformational plasticity of the MR1 binding groove $(13,28)$, the increasingly recognized diversity in MR1restricted TCRs suggests the repertoire of ligands is likely to be much broader than the vitamin B metabolites.

\section{New Classes of MR1 Ligands: Synthetic Compounds, Riboflavin-Deficient Bacteria, and Cancer Metabolism}

Inspired by the reasoning presented above, ongoing ligand identification efforts have discovered a number of non-vitamin B-derived MR1 ligands, both microbial- and non-microbial. The first non-vitamin B-derived ligands were identified through in silico modeling of putative MR1 interactions with synthetic molecules in chemical compound libraries (16). These ligands include the synthetic drug compounds diclofenac, an aspirin analog (3-formylsalicylic acid), and a methotrexate derivative (2,4-diamino-6-formylpteridine). Like the ribityllumazines and pyrimidines, these drugs are small cyclic compounds, some of which are MAIT cell agonists and some of which are antagonists (16). The role these ligands may play in drug-induced immune modulation through MAIT cell activation or inhibition is not yet clear. Using a similar in silico screen, Salio et al., recently expanded the library of MR1 ligands, including the first molecule that prevents MR1 egress from the ER (17). Intriguingly, this ligand binds in the MR1 A'-pocket in a non-covalent fashion and prevents MR1 surface translocation and MAIT cell activation in response to canonical ligands (17).

In addition to these synthetic molecules, we have found evidence for the existence of non-vitamin B metabolite microbial MR1 ligands. For example, we identified a TRAV12-2 ${ }^{+}$MR1T clone that responds to an unidentified ligand from Streptococcus pyogenes ( $S$. pyogenes), a bacterium that does not express the enzymes of the riboflavin biosynthetic pathway (10). We also performed mass spectrometry on MR1 molecules purified from cells infected with Escherichia coli (E. coli) or Mycobacterium smegmatis (M. smegmatis) (15). While the canonical ribityllumazine and pyrimidine ligands were the most common ions bound to the MR1 purified from E. coli-infected cells, these ligands had relatively low abundance in the molecules purified from $M$. smegmatis-infected cells. MR1 preparations from either infection contained ions with an ionization pattern not consistent with ribityllumazine molecules (15). Together, these data suggest a distinct class of small molecules metabolite ligands that is likely to be more prevalent in $M$. smegmatis. Consistent with this notion, Corbett et al., reported that some MR1 ligands were differentially abundant in E. coli compared to Salmonella typhimurium (S. typhimurium) (12). Together with data demonstrating the differential recognition of ligands by distinct MR1T TCRs (15), evidence that infection with different microbes drives the expansion of MR1Ts with distinct $\beta$ chains (35) further supports the idea that different bacterial species express different ligands.

Moreover, a number of studies have recently provided indirect evidence for non-microbial self-ligands for $\operatorname{MR} 1$ (9, 15, 33). In the same study describing novel microbial MR1 ligands by mass spectrometry, we also identified numerous unique ions associated with MR1 purified from uninfected insect cells. We hypothesize that some of these ions represent novel endogenous MR1 ligands, whereas others may originate from chaperones or cellular co-factors associated with MR1 loading and trafficking (15). Others have demonstrated the existence of putative selfligands in the context of tumor cell lines and primary cancers. For example, Lepore et al., identified a population of TRAV1$2^{-}$MR1Ts that recognize molecules derived from tumor cells and not microbes or cell culture medium (9). Similarly, Crowther et al. generated a non-MAIT MR1T clone specifically responding to cancer cell lines and primary cancer cells (33). Interestingly, the tumor-associated antigens reported by Lepore et al. did not form a Schiff base with MR1 like the pyrimidines and the pterins but were more similar to the ribityllumazine ligands in their non-covalent interaction with the antigen presenting molecule. The chemical identity of these MR1T antigens remains to be determined. Crowther et al. hypothesized that the ligand recognized in their system was derived from the altered metabolism characteristic of neoplastic transformation but did not report its identity. Since all remain to be identified, it is still unknown whether any of these potential self-ligands are present in healthy cells and may play a role as chaperone-like molecules such as Ii, serve as MR1T antigens that contribute to inflammation, or constitute regulatory MR1 ligands involved in immune modulation and tolerance.

\section{DISTINCT AND COMPLEMENTARY MR1 ANTIGEN PRESENTATION PATHWAYS}

\section{MR1 at Steady State: ER and Vesicular Pools but Not Much at the Cell Surface}

While MR1 has been consistently found to localize to the ER, it has also been reported to co-localize with late endosomal proteins $(30,36,37)$. We have observed a vesicular distribution of MR1 even in the absence of exogenously provided ligands (36), indicating that constitutive egress from the ER is possible. However, since endogenous MR1 is hardly detectable at the cell surface of most cell lines and primary cells, these molecules are likely very transiently expressed at the cell surface and either sequestered in intracellular stores or rapidly degraded upon internalization (38) (Figure 1, "steady state"). This hypothesis is supported by the ability of an anti-MR1 antibody to stabilize transiently expressed MR1 molecules at the cell surface (39) and the observation that MR1 detection by flow cytometry 
is cumulative when cells are incubated with antibody under conditions that allow internalization of MR1-antibody complexes (40). Of note, microbe-induced upregulation of MR1 surface expression was independent of MR1 ligand in the same study. Instead, toll-like receptor (TLR) signaling increased MR1 surface levels in some but not all antigen presenting cells and both this modulation as well as steady state MR1 surface expression were dependent on NF- $\mathrm{B}$ (40). Importantly, pre-treatment with a TLR2 agonist that induced upregulation of MR1 surface expression increased MR1-dependent antigen presentation in the same report (40), providing evidence that increased anterograde flux of MR1 could feed into an "exchange pathway" (Figure 1, discussed below). Furthermore, McWilliam et al. showed that incubation with 5-OP-RU led to the detection of a small number of MR1-5-OP-RU complexes even at $4^{\circ} \mathrm{C}$, supporting the existence of loadable MR1 molecules at the cell surface at steady state (41). MR1 requires ligand binding for stable association with $\beta_{2} \mathrm{~m}$ and acquisition of EndoH resistance, a marker of ER egress (41). Therefore, we expect that any MR1 molecules in subcellular compartments other than the ER carry a ligand. The two most likely sources of this molecule are derivatives of folate and riboflavin contained in the culture medium or an endogenous self-ligand (discussed above). Alternatively, a small proportion of ER-resident MR1 molecules may stochastically acquire a conformation that allows them to leave the ER as a result of a conformational equilibrium, as proposed by McWilliam and Villadangos based on similar concepts in MHC class Ia folding (42-44). Regardless of how different MR1 molecules reach their respective intracellular locations, the existence of ER, vesicular, and cell surface pools of MR1 conceivably contribute to the sampling of different intracellular environments harboring different sources of MR1T antigens (20). This notion is not without precedent as different pools of MHC class Ia molecules similarly survey different subcellular compartments. Specifically, nascent MHC class Ia molecules present antigens loaded in the ER whereas a subset of recycling molecules is thought to be loaded with exogenous antigen in other compartments in the context of cross-presentation [reviewed in (45)]. Notably, microscopic localization studies of MR1 so far have relied on overexpression of GFP-tagged versions of the molecule $(36,41)$. It would be extremely informative to directly investigate the intracellular distribution of endogenous MR1 but this has so far been prevented by the prohibitively low abundance of the protein in WT cells.

\section{The ER Pathway: Surface Translocation in Response to Exogenous Ligand}

The use of defined model antigens such as 5-OP-RU in both in vitro and in vivo models has enabled valuable insights into the mechanisms of MR1-mediated presentation of soluble ligands administered exogenously. These studies have elucidated an "ondemand" mode of presentation for MR1 in this context $(38,41)$. Here, MR1 resides primarily in the ER in a partially folded, ligand-receptive state. Only when exogenous ligand neutralizes the positive charge on the K43 residue in the MR1 binding groove can the protein associate with $\beta_{2} \mathrm{~m}$ and acquire the ability to leave the ER and translocate to the cell surface $(38,41)$ (Figure 1, "ER pathway"). MR1 loading in this model is thought to take place in the ER, although the mechanism by which the MR1 ligand reaches this compartment is still unclear $(22,38)$. This pathway has been the subject of excellent previous reviews to which we refer the reader [Example: (43)].

\section{The Exchange Pathway: Swapping Out Ligands on Recycling MR1 Molecules}

Recent work by our group suggests that ligand exchange plays an important role in MR1-mediated presentation of exogenous antigen (31). In this study, pre-incubation of a bronchial epithelial cell line with 6-FP overnight enhanced the presentation of exogenous ligand but did not affect MR1T activation in response to Mycobacterium tuberculosis $(M t b)$ infection. This supports a model in which pre-incubation with MR1-stabilizing ligand brings MR1 to the cell surface and from there into an exchange compartment where it can be re-loaded with exogenous antigen [(20, 22); Figure 1, "exchange pathway"]. This model is consistent with work from McWilliam et al., who showed that reloading of 6-FP-bound molecules was possible at $37^{\circ} \mathrm{C}$ but not on ice, indicating a requirement for internalization and recycling for ligand exchange to occur (41). Similarly, presentation of a set of novel MR1 ligands was reduced in cells over-expressing GPI-linked MR1 compared to those transduced with the WT protein, suggesting that a motif in the MR1 cytoplasmic tail may be required for ligand exchange and loading of some ligands (17). The notion of post-ER loading of MR1 molecules is further supported by the observation that pre-incubation with 6-FP rendered the subsequent surface expression of MR1-5-OP-RU complexes less sensitive to Brefeldin A (BFA) (41). Importantly, there was still a contribution of ER-derived molecules in this system as BFA partially reduced the MR1 surface levels (41).

Intriguingly, a shorter pre-incubation with 6-FP was previously shown to abrogate presentation of $M$. smegmatis supernatant (36), seemingly contradicting the hypothesis of an exchange pathway supplied with MR1 molecules that leave the ER bound to endogenous or exogenous antagonist ligands. These two observations could, however, be reconciled by a time-dependent model of MR1 trafficking. In this scenario, the majority of the MR1 molecules are occupied by 6-FP and localized to the cell surface after $2 \mathrm{~h}$ while overnight incubation allows enough time for internalization and recycling to the cell surface to occur. Consequently, ligand exchange is only observed upon the longer pre-incubation. McWilliam et al. reported much faster recycling kinetics for MR1, but these measurements were made in the hematopoietic cell line C1R (41) whereas our exchange studies were carried out in epithelial cells $(31,36)$. Thus, recycling kinetics might be different between cell lines, particularly since C1R cells are phagocytic professional antigen presenting cells whereas epithelial cells are not. Alternatively, the different outcomes following short compared to long 6-FP pre-incubation could be explained by the presence or absence of the antagonist during antigen presentation. Specifically, 6-FP was present for the duration of the ELISPOT after the short pre-incubation whereas the antagonist was washed off before 


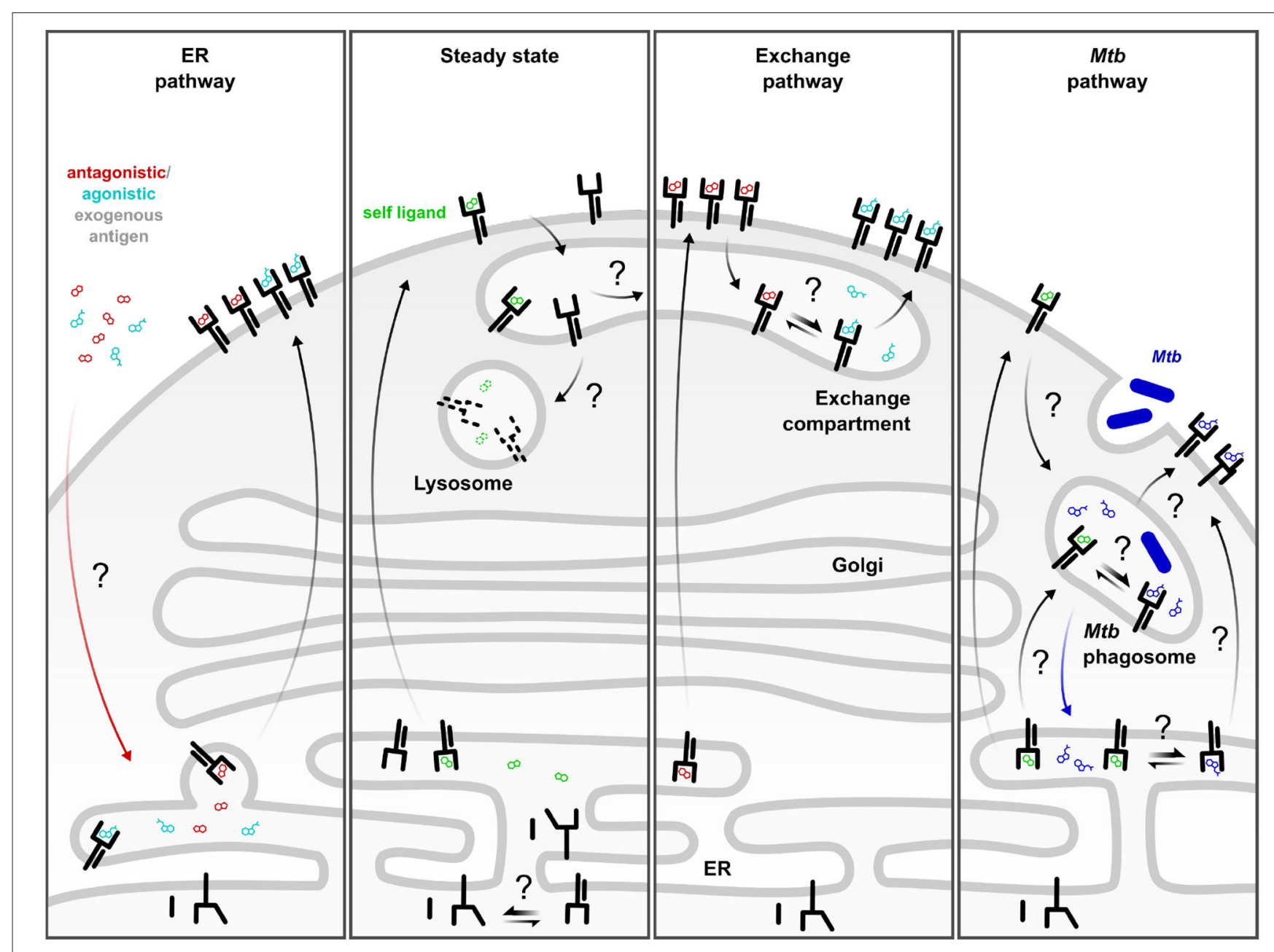

FIGURE 1 | Complementary MR1 antigen presentation pathways. ER pathway: Defined antagonistic and agonistic exogenous MR1 ligands including 6-FP and 5-OP-RU access incompletely folded MR1 in the ER and trigger its translocation to the cell surface. This pathway is dependent on neutralization of the K43 residue. Steady state: At baseline, a small fraction of MR1 molecules constitutively leaves the ER, potentially as a result of the conformational equilibrium of MR1 or through binding to an unknown self-ligand or a ubiquitous environmental ligand. These molecules are rapidly internalized and degraded in the absence of exogenous ligand or microbial infection. Exchange pathway: Alternatively, the self-ligand may be exchanged for exogenous, antigenic ligand in an exchange compartment from where the re-loaded MR1 can return to the cell surface. This process can be amplified by TLR stimulation or by pre-incubation with MR1-stabilizing antagonist ligand which increases the pool of post-ER MR1 available for exchange. Mtb pathway: Presentation of mycobacterial MR1T antigens generated upon intracellular infection are likely loaded in a Mycobacterium tuberculosis (Mtb)-specific phagosomal compartment. MR1 molecules may be delivered to the Mtb phagosome directly or via internalization from the cell surface. Alternatively, Mtb-derived ligand may reach the ER and induce MR1 surface translocation. Black arrows denote movement of MR1. Colored arrows denote movement of ligand. Straight double arrows indicate equilibria. Question marks denote hypothetical steps which have not been defined mechanistically.

co-culture with $\mathrm{T}$ cells after overnight incubation with 6-FP $(31,36)$. Thus, 6-FP was present during initial MR1 loading in both cases but was only present during presentation to MR1Ts in the $2 \mathrm{~h}$ pre-incubation experiment. While the rate of MR1 internalization from the cell surface is independent of ligand binding (41), the efficiency of ligand exchange or other aspects of MR1 antigen presentation could be differentially affected in the presence of the antagonist. Since exchange likely depends on the relative concentrations of the alternative ligands, the continuous presence of 6-FP in the first study may have shifted the equilibrium toward MR1 occupied by the antagonist.

\section{The Mtb Pathway: A Requirement for Intracellular Infection and Many Open Questions}

The intracellular pathogen $M t b$ was one of the first to be discovered to produce MR1T antigens $(18,46)$. Nevertheless, how MR1 ligands are loaded in the context of intracellular microbial infection is less well defined compared to soluble, exogenous ligands. In fact, accumulating evidence demonstrates that the molecular machinery required for the presentation of microbe-derived antigen differs from that involved in the loading of exogenous ligand $(20,22,38)$. For example, presentation 
of whole fixed E. coli bacteria is reduced upon inhibition of lysosomal acidification whereas MAIT cell activation by bacterial supernatant is not (40). In the same study, presentation of exogenous bacterial supernatant correlated with MR1 expression whereas presentation of MR1T antigen from intact bacteria was less dependent on MR1 over-expression (40). Similarly, we showed that Stx18 and VAMP4 both affect Mtb presentation but only Stx18 also affects surface translocation of MR1 in response to the stabilizing ligand 6-FP (36). This is consistent with early work by Huang et al., who showed that MR1 surface levels do not necessarily correlate with the ability to activate MAIT cells and that these two read-outs have different requirements for endosomal trafficking (47). Of note, these early experiments were carried out in the absence of bacterial infection or defined MR1T antigen.

Using transwell assays, we demonstrated that intracellular infection of the antigen presenting cell was required for the activation of MR1Ts in response to Mtb (48). By contrast, the supernatants of other bacteria such as E. coli, M. smegmatis, and $S$. pyogenes contain MR1 ligands capable of activating MR1Ts without the need for bacterial infection $(10,31,36,40)$. This difference could be explained by a comparatively low abundance of MR1 ligands produced by $M t b$. As a consequence, containment of the microbe in an endosomal compartment might be necessary to achieve sufficiently high local concentrations of the antigen for MR1 loading. As mentioned above, gene expression levels of key enzymes of riboflavin synthesis correlated with the extent of MAIT cell activation in different Streptococcus pneumoniae isolates (25). Thus, it is plausible that such differences exist at the species level also. Alternatively, Mtb may not produce secreted MR1 ligands and liberation of MR1T antigens may require endosomal processing (31). Supporting a role for intracellular infection for optimal MR1 presentation of other intracellular pathogens, Le Bourhis et al. showed that rendering Shigella flexneri incapable of invading HeLa cells drastically reduced its ability to activate MAIT cells (49). Similarly, a S. typhimurium mutant unable to actively invade non-phagocytic cells did not elicit a MAIT cell response in vivo (50). However, supernatant from this mutant still activated an MR1-restricted Jurkat T cell clone in vitro, highlighting different mechanistic requirements for in vitro presentation of exogenous antigens compared to microbial infection under physiological conditions (50). Interestingly, administration of 5-OP-RU alone was not sufficient to induce MAIT cell accumulation in murine lungs although MAIT cells were activated as measured by CD69 expression (51). Accumulation of MAIT cells in this model was dependent on TLR signaling and could be achieved by co-administration of 5-OPRU with a riboflavin-deficient bacterium or purified TLR ligands (51). This may either highlight a need for bacterial infection for optimal MR1 antigen presentation in vivo or indicate that MAIT cell expansion requires TLR-induced cytokine production at the site of infection. In another in vitro study, addition of fixed bacteria incapable of producing riboflavin did not increase MAIT cell activation in response to exogenously applied E. coli supernatant, indicating that both bacterium and ligand have to be present in the same compartment for optimal presentation in the context of MR1 in this model (40). Overall, the requirement for intracellular infection as well as the molecular mechanisms employed for antigen loading and presentation likely depend on the specific features of the infecting microbe. The metabolic state of the bacterium, the identity and stability of the MR1 ligands it produces, and the biochemical conditions it encounters in the intracellular environment are all likely determinants of the cellular mechanisms required for efficient MR1-mediated antigen presentation.

As mentioned, the requirement for intracellular infection with $M t b$ may indicate that endosomal processing is needed to generate and/or load mycobacterial antigen. While the cell surface can be a source of antigen presenting molecules that are loaded in endosomal compartments $(45,52)$, other theoretical possibilities include direct recruitment of MR1 molecules to the $M t b$ phagosome or delivery of $M t b$-derived MR1 ligands to the ER (Figure 1, "Mtb pathway"). Indeed, both classical and nonclassical MHC class I molecules have been detected in purified $M t b$ phagosomes (53). One way to account for the presence of these and other ER-resident proteins in phagosomes is the fusion of ER and phagosomal membranes $(54,55)$. Correspondingly, early evidence suggested that the ER endomembrane could contribute to phagosome membranes (54) although this has remained a point of contention [reviewed in (55)]. More recently, membrane contact sites (MCS), defined as points of close physical proximity between organelles which allow the exchange of lipids and ions without membrane fusion, have emerged as a potential explanation for the detection of ER material in phagosomal preparations (55). Alternative explanations for the presence of a subset of ER proteins in phagosomes include ER-to-phagosome vesicular trafficking and delivery of MHC-I from recycling endosomes $(45,55,56)$. Both have been extensively studied in the context of MHC class I-mediated cross-presentation and although many details remain to be elucidated, multiple studies have implicated the ER SNARE Sec22b and its interaction partner Stx 4 in the delivery of ER proteins directly to endosomal compartments $(45,57,58)$. In our hands, knock down of Sec22b resulted in reduced presentation of $M t b$-derived MR1T antigens (36) whereas Stx4 knock down specifically affected the presentation of $M$. smegmatis supernatant without inhibiting responses to $M t b$ infection (31). Thus, the extent of mechanistic overlap between MHC class I cross-presentation and MR1mediated antigen presentation remains to be determined. In fact, MR1 also associates with MHC class II chaperones under certain circumstances (47), although it is not dependent on these as evident from the observation that epithelial cells, which do not express MHC class II machinery, can present MR1T antigens $(20,36,48)$. While the MR1 antigen presentation pathway(s) may intersect with both MHC class I and class II pathways, we expect that specialized machinery exists to allow for the juxtaposition of MR1 and microbe-containing compartments and look forward to their identification.

By contrast, it is more difficult to envision a scenario in which mycobacterial antigens or even entire microbes should gain access to the ER. Although recent work by Legoux et al. implies that 5-OP-RU can not only rapidly cross lipid bilayers but even traverse skin and organs to reach the thymus when topically applied to mouse ears (59), the mechanism 
of transport remains to be identified. We hypothesize that dedicated molecular machinery is in place to capture, stabilize, and shuttle MR1 ligands between organelles and, potentially, across longer distances on micro- and macro-anatomical scales. The identification of these chaperones for MR1 ligands is of high priority for the MR1T field.

\section{OUTSTANDING QUESTIONS}

Taken together, the current literature supports a model in which redundant and complementary pathways allow MR1 to sample discrete antigens from a variety of subcellular compartments $[(20,22,38)$; Figure 1]. The chemical nature of the antigens may be critical to understanding these pathways, as different classes of ligands may be generated and presented through different pathways. The existence of previously identified neoantigens (e.g., 5-OP-RU), the observation of clusters of unidentified ligands that may represent novel neoantigens, and the evidence for self-ligands, demonstrate a need to continue working toward defining the MR1 ligandome. While there is overwhelming evidence that 5-OP-RU is a potent ligand for MR1Ts, antigens of the highest potency may not necessarily be those that are the most protective. The identification of additional ligands would also provide better tools to investigate how different sources and types of MR1T antigens relate to differential TCR recognition and clonal expansion. As such, a key outstanding question is whether and how ligand diversity contributes to memory formation. Finally, a pragmatic question regarding ligand diversity is whether ligands can be modified in order to improve stability, biosynthetic capability, bioavailability, deliverability, and other features that will be requirements if MR1Ts are to be targeted for vaccine or therapeutic development. In this respect, a number of groups have recently generated new synthetic versions of the known ligands, including glyco-analogs (60), monodeoxyribityl and monohydroxyalkyl analogs (61), and pro-drug analogs (62). A better understanding of ligand diversity will be required as work to modify ligands for therapeutic purposes moves forward.

The outstanding questions regarding MR1-mediated antigen presentation primarily center on the intracellular trafficking of both the antigen presenting molecule and its ligands. Firstly, it remains puzzling why endogenous surface levels of MR1 are extremely low, yet surface expression readily increases upon over-expression of the molecule even if cells are cultured in medium devoid of folate (41). This would indicate that ligand availability is not the only limiting factor and implicates MR1 protein abundance, too. It is tempting to speculate that there might be an active retention mechanism at play (20), similar to the extensive quality control governing the release of loaded MHC class I molecules $(63,64)$. Indeed, although it

\section{REFERENCES}

1. Godfrey DI, Uldrich AP, McCluskey J, Rossjohn J, Moody DB. The burgeoning family of unconventional T cells. Nat Immunol. (2015) 16:111423. doi: $10.1038 /$ ni.3298 has been shown that neutralization of K43 in the MR1 ligand binding groove facilitates ER egress (41), how this is detected on a molecular level is not known. Interestingly, MR1 seems to be able to breach cellular quality control mechanisms and translocate to the cell surface in a fully folded state upon incubation at $26^{\circ} \mathrm{C}$ (65). This is consistent with the idea that the molecular feature, likely a specific conformation, that releases MR1 from the ER, can be achieved without addition of exogenous ligand. We hypothesize that a key determinant of MR1 surface translocation is the extent of conformational plasticity in the heavy chain, as has been postulated in the context of peptide antigen presentation $(63,66,67)$. Neutralization of K43 may be one of multiple ways to restrict conformational flexibility in a way that enables ER egress. Related questions pertain to the stability of partially folded MR1 in the ER and how stabilizing ligands reach this compartment. Moreover, the increasing evidence for multiple presentation pathways, including the notion of ligand exchange in endosomal compartments, opens the door to numerous questions concerning the molecular mechanisms governing exchange of MR1 ligands. Since Schiff bases are more labile in acidic environments (41), one possibility is that exchange occurs simply when internalized MR1 molecules reach a point in the endocytic pathway where the $\mathrm{pH}$ is sufficiently low to destabilize the covalent bond between MR1 and its ligand. As a result, the original ligand is released, and a new ligand can be bound to "empty" MR1 molecules. In this scenario, the equilibrium between MR1 molecules bound to each ligand is determined by the $\mathrm{pH}$ of the exchange compartment and the relative concentrations of the available ligands (Figure 1, "exchange pathway"). Alternatively, exchange could be an active process catalyzed by dedicated exchange chaperones, which have been described for other MHC molecules. Examples include TAPBPR for MHC class I (64), HLA-DM for MHC class II (68), and lipid transfer proteins for CD1 molecules (69). Taken together, it is becoming clear that different MR1 antigen presentation pathways enable the MR1-MR1T axis to sample various intracellular compartments while avoiding inappropriate MR1T cell activation. The relative contributions of these complementary pathways to protective MR1T cell immunity as well as the molecular machinery underlying the individual mechanisms remain to be established.

\section{AUTHOR CONTRIBUTIONS}

$\mathrm{MH}$ and $\mathrm{CK}$ wrote and edited the manuscript. CK generated the figure. EK and DL provided intellectual contribution to the topics covered and edited the manuscript. All authors contributed to the article and approved the submitted version.

2. Lanier LL. Shades of grey-the blurring view of innate and adaptive immunity. Nat Rev Immunol. (2013) 13:73-4. doi: 10.1038/nri3389

3. Van Rhijn I, Moody DB. Donor unrestricted T cells: a shared human T cell response. J Immunol. (2015) 195:1927-32. doi: 10.4049/jimmunol.150 0943 
4. Hashimoto K, Hirai M, Kurosawa Y. A gene outside the human MHC related to classical HLA class I genes. Science. (1995) 269:693-5. doi: $10.1126 /$ science. 7624800

5. Treiner E, Duban L, Bahram S, Radosavljevic M, Wanner V, Tilloy F, et al. Selection of evolutionarily conserved mucosal-associated invariant $\mathrm{T}$ cells by MR1. Nature. (2003) 422:164-9. doi: 10.1038/nature01433

6. Godfrey DI, Koay HF, McCluskey J, Gherardin NA. The biology and functional importance of MAIT cells. Nat Immunol. (2019) 20:1110-28. doi: 10.1038/s41590-019-0444-8

7. Gherardin NA, Keller AN, Woolley RE, Le Nours J, Ritchie DS, Neeson PJ, et al. Diversity of $\mathrm{T}$ cells restricted by the MHC class i-related molecule MR1 facilitates differential antigen recognition. Immunity. (2016) 44:32-45. doi: 10.1016/j.immuni.2015.12.005

8. Koay HF, Su S, Amann-Zalcenstein D, Daley SR, Comerford I, Miosge L, et al. A divergent transcriptional landscape underpins the development and functional branching of MAIT cells. Sci Immunol. (2019) 4:eaay6039. doi: 10.1126/sciimmunol.aay6039

9. Lepore M, Kalinichenko A, Calogero S, Kumar P, Paleja B, Schmaler M, et al. Functionally diverse human $\mathrm{T}$ cells recognize non-microbial antigens presented by MR1. Elife. (2017) 6:e24476. doi: 10.7554/eLife.24476.020

10. Meermeier EW, Laugel BF, Sewell AK, Corbett AJ, Rossjohn J, McCluskey J, et al. Human TRAV1-2-negative MR1-restricted T cells detect $S$. pyogenes and alternatives to MAIT riboflavin-based antigens. Nat Commun. (2016) 7:12506. doi: $10.1038 /$ ncomms 12506

11. Tilloy F, Treiner E, Park SH, Garcia C, Lemonnier F, de la Salle H, et al. An invariant $\mathrm{T}$ cell receptor alpha chain defines a novel TAP-independent major histocompatibility complex class Ib-restricted alpha/beta T cell subpopulation in mammals. J Exp Med. (1999) 189:1907-21. doi: 10.1084/jem.189.12.1907

12. Corbett AJ, Eckle SB, Birkinshaw RW, Liu L, Patel O, Mahony J, et al. Tcell activation by transitory neo-antigens derived from distinct microbial pathways. Nature. (2014) 509:361-5. doi: 10.1038/nature13160

13. Kjer-Nielsen L, Patel O, Corbett AJ, Le Nours J, Meehan B, Liu L, et al. MR1 presents microbial vitamin B metabolites to MAIT cells. Nature. (2012) 491:717-23. doi: 10.1038/nature11605

14. Eckle SB, Corbett AJ, Keller AN, Chen Z, Godfrey DI, Liu L, et al. Recognition of vitamin B precursors and byproducts by mucosal associated invariant $\mathrm{T}$ cells. J Biol Chem. (2015) 290:30204-11. doi: 10.1074/jbc.R115.685990

15. Harriff MJ, McMurtrey C, Froyd CA, Jin H, Cansler $M$, Null $M$, et al. MR1 displays the microbial metabolome driving selective MR1restricted $\mathrm{T}$ cell receptor usage. Sci Immunol. (2018) 3:eaao2556. doi: 10.1126/sciimmunol.aao2556

16. Keller AN, Eckle SB, Xu W, Liu L, Hughes VA, Mak JY, et al. Drugs and druglike molecules can modulate the function of mucosal-associated invariant $\mathrm{T}$ cells. Nat Immunol. (2017) 18:402-11. doi: 10.1038/ni.3679

17. Salio M, Awad W, Veerapen N, Gonzalez-Lopez C, Kulicke C, Waithe D, et al. Ligand-dependent downregulation of MR1 cell surface expression. Proc Natl Acad Sci USA. (2020) 117:10465-75. doi: 10.1073/pnas.2003136117

18. Le Bourhis L, Martin E, Peguillet I, Guihot A, Froux N, Core M, et al. Antimicrobial activity of mucosal-associated invariant T cells. Nat Immunol. (2010) 11:701-8. doi: 10.1038/ni.1890

19. Tastan C, Karhan E, Zhou W, Fleming E, Voigt AY, Yao X, et al. Tuning of human MAIT cell activation by commensal bacteria species and MR1-dependent T-cell presentation. Mucosal Immunol. (2018) 11:1591-605. doi: 10.1038/s41385-018-0072-x

20. Karamooz E, Harriff MJ, Lewinsohn DM. MR1-dependent antigen presentation. Semin Cell Dev Biol. (2018) 84:58-64. doi: 10.1016/j.semcdb.2017.11.028

21. Rock KL, Reits E, Neefjes J. Present yourself! By MHC class I and MHC class II molecules. Trends Immunol. (2016) 37:724-37. doi: 10.1016/j.it.2016.08.010

22. Lamichhane R, Ussher JE. Expression and trafficking of MR1. Immunology. (2017) 151:270-9. doi: 10.1111/imm.12744

23. Eckle SB, Birkinshaw RW, Kostenko L, Corbett AJ, McWilliam HE, Reantragoon $\mathrm{R}$, et al. A molecular basis underpinning the $\mathrm{T}$ cell receptor heterogeneity of mucosal-associated invariant T cells. J Exp Med. (2014) 211:1585-600. doi: 10.1084/jem.20140484

24. Patel O, Kjer-Nielsen L, Le Nours J, Eckle SB, Birkinshaw R, Beddoe T, et al. Recognition of vitamin B metabolites by mucosal-associated invariant $\mathrm{T}$ cells. Nat Commun. (2013) 4:2142. doi: 10.1038/ncomms3142
25. Hartmann N, McMurtrey C, Sorensen ML, Huber ME, Kurapova R, Coleman FT, et al. Riboflavin metabolism variation among clinical isolates of streptococcus pneumoniae results in differential activation of mucosalassociated invariant T cells. Am J Respir Cell Mol Biol. (2018) 58:767-76. doi: 10.1165/rcmb.2017-0290OC

26. Soudais C, Samassa F, Sarkis M, Le Bourhis L, Bessoles S, Blanot D, et al. In vitro and in vivo analysis of the gram-negative bacteria-derived riboflavin precursor derivatives activating mouse MAIT cells. J Immunol. (2015) 194:4641-9. doi: 10.4049/jimmunol.1403224

27. Yamaguchi H, Hashimoto K. Association of MR1 protein, an MHC class Irelated molecule, with beta(2)-microglobulin. Biochem Biophys Res Commun. (2002) 290:722-9. doi: 10.1006/bbrc.2001.6277

28. Lopez-Sagaseta J, Dulberger CL, Crooks JE, Parks CD, Luoma AM, McFedries $\mathrm{A}$, et al. The molecular basis for mucosal-associated invariant $\mathrm{T}$ cell recognition of MR1 proteins. Proc Natl Acad Sci USA. (2013) 110:E1771-8. doi: $10.1073 /$ pnas. 1222678110

29. Lopez-Sagaseta J, Dulberger CL, McFedries A, Cushman M, Saghatelian A, Adams EJ. MAIT recognition of a stimulatory bacterial antigen bound to MR1. J Immunol. (2013) 191:5268-77. doi: 10.4049/jimmunol.1301958

30. McWilliam HE, Birkinshaw RW, Villadangos JA, McCluskey J, Rossjohn J. MR1 presentation of vitamin B-based metabolite ligands. Curr Opin Immunol. (2015) 34:28-34. doi: 10.1016/j.coi.2014.12.004

31. Karamooz E, Harriff MJ, Narayanan GA, Worley A, Lewinsohn DM. MR1 recycling and blockade of endosomal trafficking reveal distinguishable antigen presentation pathways between Mycobacterium tuberculosis infection and exogenously delivered antigens. Sci Rep. (2019) 9:4797. doi: 10.1038/s41598-019-41402-y

32. Gherardin NA, McCluskey J, Rossjohn J, Godfrey DI. The diverse family of MR1-restricted T cells. J Immunol. (2018) 201:2862-71. doi: 10.4049/jimmunol.1801091

33. Crowther MD, Dolton G, Legut M, Caillaud ME, Lloyd A, Attaf M, et al. Genome-wide CRISPR-Cas9 screening reveals ubiquitous $\mathrm{T}$ cell cancer targeting via the monomorphic MHC class I-related protein MR1. Nat Immunol. (2020) 21:178-85. doi: 10.1038/s41590-019-0578-8

34. Gold MC, McLaren JE, Reistetter JA, Smyk-Pearson S, Ladell K, Swarbrick GM, et al. MR1-restricted MAIT cells display ligand discrimination and pathogen selectivity through distinct T cell receptor usage. J Exp Med. (2014) 211:1601-10. doi: 10.1084/jem.20140507

35. Gold MC, Eid T, Smyk-Pearson S, Eberling Y, Swarbrick GM, Langley SM, et al. Human thymic MR1-restricted MAIT cells are innate pathogen-reactive effectors that adapt following thymic egress. Mucosal Immunol. (2013) 6:3544. doi: $10.1038 / \mathrm{mi} .2012 .45$

36. Harriff MJ, Karamooz E, Burr A, Grant WF, Canfield ET, Sorensen ML, et al. Endosomal MR1 trafficking plays a key role in presentation of Mycobacterium tuberculosis ligands to MAIT cells. PLoS Pathog. (2016) 12:e1005524. doi: 10.1371/journal.ppat.1005524

37. Huang W, He W, Shi X, He X, Dou L, Gao Y. The role of CD1d and MR1 restricted T cells in the liver. Front Immunol. (2018) 9:2424 doi: 10.3389/fimmu.2018.02424

38. McWilliam HE, Villadangos JA. MR1 antigen presentation to MAIT cells: new ligands, diverse pathways? Curr Opin Immunol. (2018) 52:108-13. doi: 10.1016/j.coi.2018.04.022

39. Chua WJ, Kim S, Myers N, Huang S, Yu L, Fremont DH, et al. Endogenous MHC-related protein 1 is transiently expressed on the plasma membrane in a conformation that activates mucosal-associated invariant T cells. J Immunol. (2011) 186:4744-50. doi: 10.4049/jimmunol.100 3254

40. Ussher JE, van Wilgenburg B, Hannaway RF, Ruustal K, Phalora P, Kurioka A, et al. TLR signaling in human antigen-presenting cells regulates MR1dependent activation of MAIT cells. Eur J Immunol. (2016) 46:1600-14. doi: 10.1002/eji.201545969

41. McWilliam HE, Eckle SB, Theodossis A, Liu L, Chen Z, Wubben JM, et al. The intracellular pathway for the presentation of vitamin B-related antigens by the antigen-presenting molecule MR1. Nat Immunol. (2016) 17:531-7. doi: $10.1038 /$ ni.3416

42. Elliott $\mathrm{T}$, Cerundolo V, Elvin J, Townsend A. Peptide-induced conformational change of the class I heavy chain. Nature. (1991) 351:402-6. doi: $10.1038 / 351402 \mathrm{a} 0$ 
43. McWilliam HEG, Villadangos JA. How MR1 presents a pathogen metabolic signature to mucosal-associated invariant T (MAIT) cells. Trends Immunol. (2017) 38:679-89. doi: 10.1016/j.it.2017.06.005

44. Townsend A, Elliott T, Cerundolo V, Foster L, Barber B, Tse A. Assembly of MHC class I molecules analyzed in vitro. Cell. (1990) 62:285-95. doi: 10.1016/0092-8674(90)90366-M

45. Blander JM. Regulation of the cell biology of antigen cross-presentation. Annu Rev Immunol. (2018) 36:717-53. doi: 10.1146/annurev-immunol-041015-055523

46. Gold MC, Cerri S, Smyk-Pearson S, Cansler ME, Vogt TM, Delepine J, et al. Human mucosal associated invariant $\mathrm{T}$ cells detect bacterially infected cells. PLoS Biol. (2010) 8:e1000407. doi: 10.1371/journal.pbio.1000407

47. Huang S, Gilfillan S, Kim S, Thompson B, Wang X, Sant AJ, et al. MR1 uses an endocytic pathway to activate mucosal-associated invariant T cells. J Exp Med. (2008) 205:1201-11. doi: 10.1084/jem.20072579

48. Harriff MJ, Cansler ME, Toren KG, Canfield ET, Kwak S, Gold MC, et al. Human lung epithelial cells contain Mycobacterium tuberculosis in a late endosomal vacuole and are efficiently recognized by CD8(+) T cells. PLoS ONE. (2014) 9:e97515. doi: 10.1371/journal.pone.0097515

49. Le Bourhis L, Dusseaux M, Bohineust A, Bessoles S, Martin E, Premel V, et al. MAIT cells detect and efficiently lyse bacterially-infected epithelial cells. PLoS Pathog. (2013) 9:e1003681. doi: 10.1371/journal.ppat.1003681

50. Wang H, Kjer-Nielsen L, Shi M, DSouza C, Pediongco TJ, Cao H, et al. IL-23 costimulates antigen-specific MAIT cell activation and enables vaccination against bacterial infection. Sci Immunol. (2019) 4:eaaw0402. doi: 10.1126/sciimmunol.aaw0402

51. Chen Z, Wang H, DSouza C, Sun S, Kostenko L, Eckle SB, et al. Mucosalassociated invariant $\mathrm{T}$-cell activation and accumulation after in vivo infection depends on microbial riboflavin synthesis and co-stimulatory signals. Mucosal Immunol. (2017) 10:58-68. doi: 10.1038/mi.2016.39

52. Moody DB, Cotton RN. Four pathways of CD1 antigen presentation to T cells. Curr Opin Immunol. (2017) 46:127-33. doi: 10.1016/j.coi.2017.07.013

53. Grotzke JE, Harriff MJ, Siler AC, Nolt D, Delepine J, Lewinsohn DA, et al. The Mycobacterium tuberculosis phagosome is a HLA-I processing competent organelle. PLoS Pathog. (2009) 5:e1000374. doi: 10.1371/journal.ppat.1000374

54. Gagnon E, Duclos S, Rondeau C, Chevet E, Cameron PH, SteeleMortimer $\mathrm{O}$, et al. Endoplasmic reticulum-mediated phagocytosis is a mechanism of entry into macrophages. Cell. (2002) 110:119-31. doi: 10.1016/S0092-8674(02)00797-3

55. Levin-Konigsberg R, Grinstein S. Phagosome-endoplasmic reticulum contacts: kissing and not running. Traffic. (2020) 21:172-80. doi: $10.1111 /$ tra. 12708

56. Montealegre S, van Endert PM. Endocytic recycling of MHC class I molecules in non-professional antigen presenting and dendritic cells. Front Immunol. (2018) 9:3098. doi: 10.3389/fimmu.2018.03098

57. Cebrian I, Visentin G, Blanchard N, Jouve M, Bobard A, Moita C, et al. Sec22b regulates phagosomal maturation and antigen crosspresentation by dendritic cells. Cell. (2011) 147:1355-68. doi: 10.1016/j.cell.2011.11.021

58. Nair-Gupta P, Baccarini A, Tung N, Seyffer F, Florey O, Huang Y, et al. TLR signals induce phagosomal MHC-I delivery from the endosomal recycling compartment to allow cross-presentation. Cell. (2014) 158:506-21. doi: 10.1016/j.cell.2014.04.054
59. Legoux F, Bellet D, Daviaud C, El Morr Y, Darbois A, Niort K, et al. Microbial metabolites control the thymic development of mucosal-associated invariant T cells. Science. (2019) 366:494-9. doi: 10.1126/science.aaw2719

60. Braganza CD, Shibata K, Fujiwara A, Motozono C, Sonoda KH, Yamasaki $\mathrm{S}$, et al. The effect of MR1 ligand glyco-analogues on mucosal-associated invariant T. (MAIT) cell activation. Org Biomol Chem. (2019) 17:8992-9000. doi: 10.1039/C9OB01436E

61. Ler GJM, Xu W, Mak JYW, Liu L, Bernhardt PV, Fairlie DP. Computer modelling and synthesis of deoxy and monohydroxy analogues of a ribitylaminouracil bacterial metabolite that potently activates human $\mathrm{T}$ cells. Chemistry. (2019) 25:15594-608. doi: 10.1002/chem.201903732

62. Lange J, Anderson RJ, Marshall AJ, Chan STS, Bilbrough TS, Gasser O, et al. The chemical synthesis, stability, and activity of MAIT cell prodrug agonists that access MR1 in recycling endosomes. ACS Chem Biol. (2020) 15:437-45. doi: 10.1021/acschembio.9b00902

63. Springer S. Transport and quality control of MHC class I molecules in the early secretory pathway. Curr Opin Immunol. (2015) 34:83-90. doi: 10.1016/j.coi.2015.02.009

64. Thomas C, Tampe R. MHC I chaperone complexes shaping immunity. Curr Opin Immunol. (2019) 58:9-15. doi: 10.1016/j.coi.2019.01.001

65. Abos B, Gomez Del Moral M, Gozalbo-Lopez B, Lopez-Relano J, Viana V, Martinez-Naves E. Human MR1 expression on the cell surface is acid sensitive, proteasome independent and increases after culturing at 26 degrees C. Biochem Biophys Res Commun. (2011) 411:632-6. doi: 10.1016/j.bbrc.2011.07.007

66. Hein Z, Uchtenhagen H, Abualrous ET, Saini SK, Janssen L, Van Hateren A, et al. Peptide-independent stabilization of MHC class I molecules breaches cellular quality control. J Cell Sci. (2014) 127:2885-97. doi: 10.1242/jcs.1 45334

67. Wieczorek M, Abualrous ET, Sticht J, Alvaro-Benito M, Stolzenberg S, Noe F, et al. Major histocompatibility complex (MHC) class I and MHC class II proteins: conformational plasticity in antigen presentation. Front Immunol. (2017) 8:292. doi: 10.3389/fimmu.2017.00292

68. Mellins ED, Stern LJ. HLA-DM and HLA-DO, key regulators of MHCII processing and presentation. Curr Opin Immunol. (2014) 26:115-22. doi: 10.1016/j.coi.2013.11.005

69. Teyton L. Role of lipid transfer proteins in loading CD1 antigen-presenting molecules. J Lipid Res. (2018) 59:1367-73. doi: 10.1194/jlr.R083212

Conflict of Interest: The authors declare that the research was conducted in the absence of any commercial or financial relationships that could be construed as a potential conflict of interest.

The handling editor declared a past co-authorship with one of the authors DL.

Copyright (C) 2020 Kulicke, Karamooz, Lewinsohn and Harriff. This is an open-access article distributed under the terms of the Creative Commons Attribution License (CC $B Y)$. The use, distribution or reproduction in other forums is permitted, provided the original author(s) and the copyright owner(s) are credited and that the original publication in this journal is cited, in accordance with accepted academic practice. No use, distribution or reproduction is permitted which does not comply with these terms. 